\title{
Del colonialismo y otros demonios: fenicios en el sur peninsular entre los siglos IX y VII/VI a.C.
}

\author{
On colonialism and other demons. Phoenicians in the South of the \\ Iberian Peninsula between the $9^{\text {th }}$ and $7^{\text {th }} / 6^{\text {th }}$ centuries B.C.
}

\author{
Beatriz Marín Aguilera \\ Departamento de Prehistoria \\ Universidad Complutense de Madrid \\ Avda. Profesor Aranguren, s/n, 28040 Madrid \\ beatriz.marin@ghis.ucm.es
}

Recibido: 18-07-2012

Aceptado: 20-09-2012

\begin{abstract}
RESUMEN
A finales del siglo IX a.C., los fenicios fundaron asentamientos permanentes a lo largo del Mediterráneo. El objetivo de este estudio es analizar no tanto dichos asentamientos como la influencia que ha ejercido el colonialismo europeo en el modo en el cual comprendemos el contacto fenicio con las poblaciones locales. Como la arqueología del Mediterráneo ha dedicado más atención a los colonizadores que a las relaciones establecidas con la gente que vivía en esos territorios, este artículo comienza con un análisis de la terminología colonial para evaluar sus raíces y el impacto del imperialismo europeo en conceptos como "colonia" y periodo "orientalizante". Esta discusión va seguida del examen de las posturas poscoloniales sobre las situaciones de contacto cultural a través del registro arqueológico en el Sur ibérico entre los siglos IX y VII/VI a.C. Finalmente, el artículo analiza las relaciones entre los fenicios y las poblaciones locales en el mediodía peninsular, cuestionando el "colonialismo" fenicio durante ese periodo.
\end{abstract}

Palabras Clave: Colonia. Civilización. Periodo orientalizante. Teoría poscolonial. Colonialismo.

\begin{abstract}
By the end of the 9 th century BC, Phoenicians set up permanent settlements around the Mediterranean Basin. This study aims at discussing how European colonialism has influenced the way in which we understand Phoenician contact with native populations. Because Mediterranean archaeology has always paid more attention to the colonizers than to the relationships established with the people who lived in those territories, this paper starts with an analysis of colonial terminology to evaluate its roots and the impact of European imperialism in concepts such as "colony" and "orientalizing" period. This is followed by an examination of postcolonial views of cultural contact situations through material culture in South Iberia between the 9th-7/6th centuries BC. The paper culminates with an appraisal of the relationship between Phoenicians and the inhabitants of South Iberia, questioning Phoenician "colonialism" during that period.
\end{abstract}

KeY words: Colony. Civilisation. Orientalizing period. Postcolonial theory. Colonialism.

Sumario: 1. Introducción. 2. Sobre la terminología y su semántica. 3. La sombra del colonialismo europeo es alargada. 4. Imaginarios coloniales y respuestas locales: el colonialismo fenicio en cuestión. 5. Consideraciones finales. 


\section{Introducción}

Contaba Diodoro de Sicilia que "los fenicios, que, desde una época lejana, navegaban sin cesar para hacer comercio, habían fundado muchas colonias sobre las costas de Libia y un cierto número de otras en las regiones occidentales de Europa" (V, 20). Esta imagen clásica está asociada a los diferentes materiales y asentamientos fenicios repartidos por las costas mediterráneas desde el siglo IX al VII/VI a.C. (Fig. 1).

La importancia de la cultura oriental, sin embargo, ha sido negada o minusvalorada en relación a la griega, y el colonialismo fenicio ha sido entendido dentro de los mismos parámetros que el griego, sin tener en cuenta las diferencias entre ambos hasta fechas recientes (cfr. Niemeyer 1990; van Dommelen 1997a; 1998). Esta preponderancia del colonialismo griego (y después del romano) frente al fenicio (y el cartaginés) viene determinada por las construcciones coloniales y nacionales/regionales de lo que es Europa y de lo que no es, es decir, de lo que significa la civilización y de lo que significa la barbarie (Morris 1994; Shanks 1996; Abdel-Malek 1963; Said 2002).

A este respecto, cabe indicar que la misión civilizadora en las colonias durante los siglos XIX y XX estuvo fuertemente enraizada en la tradición clásica, esto es, en las culturas de Grecia y Roma, herederos de las mismas como se sentían ingleses, franceses y alemanes, en detrimento de sus contemporáneos griegos e italianos (Morris 1994; Shanks 1996: 53-91; van Dommelen 1997a).
El ensalzamiento de las culturas griega y romana como señas de identidad de los europeos y como modelo para sus conquistas exteriores, favoreció los estudios sobre las colonizaciones griega y romana en términos positivos para las poblaciones de aquellos lugares donde se asentaron, de tal manera que la "helenización" y la "romanización" -y más tardíamente, la "orientalización" o el "período orientalizante", en relación a la colonización fenicia-, fueron asociadas con la "europeización" de las colonias del siglo XIX y XX (Hingley 2000; Mattingly 2011).

La analogía entre las colonias antiguas y las europeas ha sido de tal magnitud, que muchos investigadores se han llegado a preguntar hasta qué punto es pertinente aplicar la terminología colonial "moderna" al pasado mediterráneo (Malkin 2004; Owen 2005; van Dommelen 2005; De Angelis 2009). Colonia, colonización y/o colonialismo, así como "orientalizante", son términos que arrastran una tradición colonial/imperial muy fuerte tras de sí, de tal forma que el análisis de sus raíces históricas es clave para comprender qué subyace detrás de cada término utilizado en el estudio de los asentamientos fenicios, griegos y romanos.

El énfasis, no obstante, en el proceso colonial y en los colonizadores, ha silenciado o mermado sustancialmente las relaciones con y las respuestas de las poblaciones que habitaban las tierras antes de la llegada de los colonos (Boardman 1980; Blázquez 1992; Ridgway 1992; Aubet 1997); hecho derivado igualmente del colonialismo de los siglos XIX y XX, que silenciaba a los colonizados como una

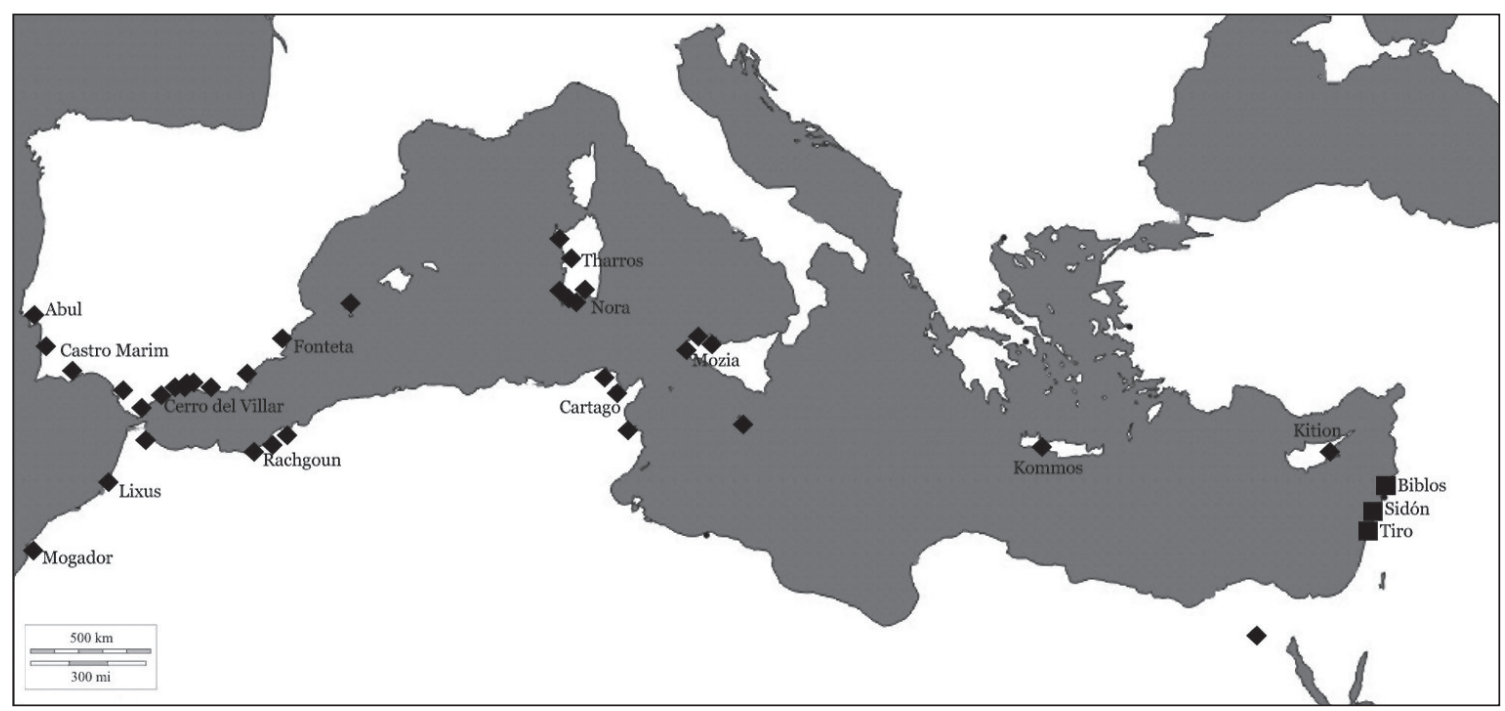

Figura 1.- Mapa de los asentamientos fenicios en el Mediterráneo durante los siglos IX-VI a.C. 
estrategia más de violencia colonial/imperial. Estudios recientes en el sur peninsular, sin embargo, traslucen un panorama bastante diverso en cuanto a las relaciones establecidas entre la población local y las colonias fenicias de la costa, que cuestiona la narración de dominación colonial que prevalece en los trabajos sobre la presencia fenicia (Ruiz Mata y González Rodríguez 1994; López Pardo y Suárez Padilla 2003).

De este modo, en el presente trabajo se analiza, primero, la influencia del colonialismo europeo y de la "misión civilizadora" en términos como "helenización", "romanización" y "orientalizante", con especial énfasis en éste último debido a su relación con la presencia fenicia en el Mediterráneo. La importancia de la terminología nos lleva al análisis semántico de las palabras usadas para definir los asentamientos fenicios en el sur peninsular, tanto de origen griego, como apoikia, como de origen romano, como colonia, así como la definición de colonialismo. Finalmente, dentro del marco teórico poscolonial, se cuestionan los modelos de dominación y explotación fenicios, es decir, el propio "colonialismo" fenicio en el sur peninsular entre los siglos IX y VII/VI a.C.

\section{Sobre la terminología y su semántica}

La Arqueología nació en el siglo XIX, ligada a dos procesos contemporáneos, el colonialismo fuera de las fronteras europeas (Morris 1994; Shanks 1996), y el desarrollo del nacionalismo en los países europeos (Gillis 1994; Evans y Boswell 1999). Imbricada en ambos proyectos, uno externo y otro interno, la arqueología sirvió a ambos intereses para la construcción de un discurso de identidad nacional por un lado (Leoussi 1998; Hingley 2001), y de identidad europea frente a los Otros (sensu Lacan), por otro (Abdel-Malek 1963; Said 2002).

En la base de ambos proyectos se situaba el discurso de la "civilización", centrado en el pasado mediterráneo griego y romano, que fue idealizado por Alemania, Francia, Inglaterra e, incluso, Estados Unidos para la construcción de su historia nacional y colonial (Johnson 1988; Friedman 1992; Richard 1995; van Dommelen 1998: 22-24). De este modo, se articuló una identidad griega y romana hegemónica, supuestamente heredada por Europa, a través de la creación de imágenes opuestas, es decir, del bárbaro y del salvaje (Hall 1989; Jiménez Díez 2008: 25-26), representado éste último por el mundo oriental. El sujeto "oriental" era así visto como un ser irracional, depravado e infantil, frente a un europeo racional, virtuoso y maduro, quedando el primero alienado en favor de las manipulaciones coloniales de Occidente (Abdel-Malek 1963; Said 2002: 68-69).

Como consecuencia, la aportación fenicia ha estado en términos generales subordinada a la presencia griega hasta fechas recientes, al igual que ha sucedido con la cartaginesa con respecto de la romana (Arteaga Matute 1995 con bibliografía anterior; Vella 1996 con bibliografía anterior). A partir de los años 80, sin embargo, el estudio de la colonización fenicia adquirió mayor relevancia en la Academia, si bien es cierto que el enfoque se centró casi exclusivamente en los colonizadores fenicios (excluyendo los estudios autoctonistas sobre los tartesios), con escasas o ninguna referencia a las poblaciones indígenas y a las consecuentes relaciones entre ambos hasta fechas recientes $(v$. Aubet 1997).

\subsection{El "orientalizante" y sus raíces coloniales}

Esta importancia del colonizador en los estudios sobre el colonialismo antiguo viene definida por la expansión de la "civilización europea" moderna y de sus valores en las colonias (Friedman 1992; Mattingly 1996; van Dommelen 1998: 18-23; Hingley 2001). La base del proyecto imperialista europeo asumía que era el colonizador el que portaba y extendía la "cultura" y que, por tanto, los colonizados no aportaban nada porque eran "bárbaros" e inferiores (Spivak 1990: 1).

Es en este contexto cuando se insertaron términos como "helenización" y "romanización", profundamente asociados con el imperialismo y con la "misión civilizadora" de los europeos en las colonias (Friedman 1990; Mattingly 2011). Dichas nociones, de factura imperial, implicaban (e implican) que el cambio cultural era unidireccional y unilineal, de las culturas consideradas como "superiores" -las colonizadoras-, a las "inferiores" -las comunidades receptoras-, cristalizando así el evolucionismo dentro de las tesis difusionistas en arqueología. El cambio cultural respondía, en este sentido, a un agente externo y no a un proceso interno, donde el colonizador cumplía el papel de "modernizar" y de "civilizar" a sociedades "retrasadas" y "bárbaras" (Woolf 1997).

Este tipo de términos, además, potenciaron (y potencian) los estudios sobre la élite y sobre los colonizadores, en vez de los análisis en profundidad de la sociedad y de las relaciones que pudieron generarse, favoreciendo así el enfoque pro-heleno/ romano en detrimento de la población autóctona (Mattingly 2011: 38-39). Al enfatizar de forma desmesurada las "gracias" de los colonizadores y la importancia de la élite, las nociones de "heleni- 
zación" y de "romanización" continúan reforzando interpretaciones culturales simplistas, como los discursos de aculturación y de emulación, y silencian los aspectos continuistas de las comunidades locales (Mattingly 2011: 39).

Bajo la misma concepción colonial/imperial surge el término "orientalizante", que es a la presencia fenicia lo que el término "helenización" a la griega y el de "romanización" a la colonización/ conquista romana. Acuñado por los historiadores del arte para hacer referencia a los objetos y técnicas de estilo oriental encontrados en contextos indígenas repartidos por todo el Mediterráneo, el concepto de "orientalizante" pasó rápidamente del hecho puramente descriptivo al interpretativo, esto es, a situarse como eje del cambio cultural de las poblaciones locales en la primera mitad del primer milenio a.C. (Aubet 2005; Purcell 2006).

En este sentido, al igual que en el caso de las culturas griega y romana, la cultura fenicia era considerada como "superior" a la que caracterizaba a las poblaciones nativas, de forma que han sido los objetos y estructuras de origen oriental los que han ocupado el interés por parte de los investigadores, que se han centrado en la apropiación de los mismos por parte de la élite indígena, según fenómenos de aculturación, (simple) imitación o emulación (Aguayo et al. 1991; Almagro Gorbea 1991; Blázquez 1992; Torres Ortiz 2002; Jiménez Ávila 2002; Aubet 2005). De este modo, la influencia fenicia ha sido magnificada y, por contra, el resto de la sociedad local ha sido silenciada, así como los procesos de continuidad de la mayoría de las poblaciones del sur peninsular.

Si algo caracteriza la investigación sobre el “orientalizante" peninsular es la nula reflexión en torno a la terminología empleada y a su origen colonial/imperial. Resulta cuanto menos sorprendente, dada la profusión de textos sobre el "orientalizante" peninsular, la escasa crítica llevada a cabo por los investigadores de dicho período que, cuando la han efectuado, se han centrado en la crítica a los modelos difusionistas inherentes al período "orientalizante" (González Wagner 1993), y en la excesiva importancia dada a la colonización fenicia sin explicar la complejidad de los procesos internos vividos por las comunidades locales (Aubet 2005; Celestino Pérez 2008: 128-131).

A pesar de las críticas a los términos "helenización" y "romanización", de fuerte raigambre colonial (Friedman 1990; Hodos 2006: 11-16; Mattingly 2011: 38-40), el colonialismo del término "orientalizante" continúa intacto, aplicándose de forma irreflexiva por la gran mayoría de los autores (v. Ruiz Mata y Celestino Pérez 2001; Celestino Pérez y Jiménez Ávila 2005).
El continuo uso de los términos "helenización", "romanización" y "orientalizante" es constitutivo de la colonialidad [sic] del saber (Grosfoguel 2007; Mignolo 2008), que tras la descolonización jurídico-política de las colonias, sigue reflejando el discurso de superioridad europeo basado en el uso y la manipulación del pasado mediterráneo, donde el discurso de "civilización" es central para legitimar el papel de las potencias europeas tanto en la época colonial como en el presente, ahora bajo el eslogan de la "cooperación" (Grosfoguel 2007; González Ruibal 2010). A este respecto, se hace necesaria la supresión de términos como "orientalizante" en la investigación sobre la presencia oriental en el Mediterráneo y el cuestionamiento de otros como "colonia" y "colonialismo", así como la pertinencia de su aplicación a los contextos fenicios entre los siglos IX y VI a.C., para conseguir un verdadero desprendimiento epistémico que elimine la linealidad de la Historia europea y la justificación de la empresa colonial en términos arqueológicos/históricos (pace González Ruibal 2008).

\subsection{Colonias, puertos de comercio y colonialismo}

Como consecuencia de la adulteración de las culturas griega y romana para inscribirlas en los discursos de "civilización" europeos, la presencia griega $\mathrm{y}$, sobre todo, la conquista romana, han favorecido una idea de lo que es una colonia y de lo que significa el colonialismo lejos de la realidad de otros contextos, como el oriental. Con ello, el colonialismo fenicio ha sido entendido dentro de los mismos parámetros que los colonialismos griego y romano, usando en el primer caso los términos $\dot{\alpha} \pi$ oıkí $\alpha$

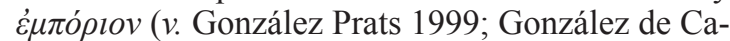
nales et al. 2004); y en el segundo, el término romano colonia (v. González Wagner 1993; Aubet 1997; Aubet y Delgado Hervás 2003). Así pues, el análisis del significado de dichos términos es clave para comprender lo que subyace detrás de los mismos.

En términos semánticos, apoikia ("d̉oıkía") hace referencia a una "casa fuera de casa", y deriva del verbo apoikipso, que significa alejarse o establecerse como colono, del que también deriva la palabra apoikos, es decir, alguien alejado de su tierra, emigrado. Para hacer referencia al comercio, los griegos no usaban la palabra apoikia, sino emporion

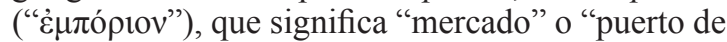
comercio". La suma de estas comunidades dispersas geográficamente era denominada oikouméne

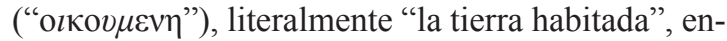
fatizando la conexión entre la ciudad de origen y la creada ex novo en otros lugares por los colonos (Malkin 2003; Hodos 2006: 19-22). 
En el caso romano, el término colonia tiene su origen en la palabra latina colōnia, que significa "propiedad rural, labriego, o colonia", haciendo hincapié en la base agrícola de estos asentamientos nuevos, pues deriva del verbo colére, "cultivar", de forma que la palabra colonus puede ser traducida indistintamente como "colono" y como "campesino" (Bispham 2006: 76-78). El establecimiento de colonias por parte de los romanos implicaba una lejanía de su lugar de origen, pero también, y más importante, una reorganización y redistribución de las tierras en relación con una conquista y explotación militar (Osborne 2008: 281; van Dommelen 2012: 396).

Las diferencias entre la terminología y, por tanto, entre la concepción "colonial" griega y romana son notables, pues la primera no implicó en ningún caso la conquista militar (aunque pudiese implicar otros modos de violencia), lo que ha llevado a muchos autores a cuestionar la naturaleza colonial de los asentamientos griegos de los siglos VIII, VII y VI a.C. (van Dommelen 1997b; Osborne 1998, 2008; Gosden 2004: 69), así como el uso de la terminología romana-europea para definir la presencia fenicia y griega en el Mediterráneo entre los siglos VIII y VI a.C. (Malkin 2004; Owen 2005; van Dommelen 2005; De Angelis 2009).

A pesar de ello, nuestros conceptos de "colonia" y de "colonialismo", así como su comprensión, derivan del latín y del colonialismo romano y, sobre todo, del colonialismo europeo. No es coincidencia, en este sentido, que el término colonia hiciese su aparición en los diccionarios con el significado de "colonia de personas enviada a habitar otro territorio conseguido por conquista" sólo a partir del siglo XVI, en clara relación con el colonialismo español en América (Alvar Ezquerra y Nieto Jiménez 2007: 2671).

Así pues, el colonialismo, aunque ha tenido diferentes significados dependiendo de la época y de las diferentes tradiciones en las que se ha visto inmerso, puede definirse como el proceso de establecimiento de grupos de población lejos de su lugar de origen y la existencia de relaciones asimétricas de explotación y dominación entre colonizadores y colonizados (Horvath 1972; Said 1996: 43; van Dommelen 1998: 16; Rowlands 1998). A esta definición habría que añadir, además, la migración de un número considerable de personas a los territorios conquistados/colonizados, proceso al que pocas veces se presta atención (van Dommelen 2012). Esta definición de colonialismo, marcada por la huella romana y por un pasado europeo mucho más reciente, deja fuera, sin embargo, los asentamientos fenicios del siglo IX al VI a.C. (vid. Infra), que responden a una migración escasa y no a una conquista militar ni a una idea imperial insertada dentro de un movimiento colonizador cuyo objetivo es controlar las regiones donde se establecen (Stein 2002: 29; Osborne 2008: 281-282; cfr. Moreno Arrastio 1999; González Wagner 2007; González Ruibal 2008).

\section{La sombra del colonialismo europeo es alargada}

La notable influencia que ha tenido el colonialismo europeo y su "misión civilizadora" en la comprensión y definición de la presencia fenicia, griega y romana en el Mediterráneo es parte inherente del discurso de "civilización" del que se hablaba al principio de este trabajo. Este discurso, base de las atrocidades del colonialismo europeo, fue criticado con dureza desde 1927 en adelante con la creación de la Liga contra el imperialismo y la opresión colonial en Bruselas, a la que siguieron nuevas conferencias e iniciativas que unieron a pensadores como Amílcar Cabral, Leopold Sédar Senghor y Albert Memmi, de África; Mahatma Gandhi y Ali Shariati de Asia; y Aimé Césaire, José Carlos Mariátegui y Frantz Fanon de Latinoamérica y el Caribe, entre otros.

Heredera de estos autores, la teoría poscolonial adquiere cuerpo en 1978 con la famosa obra del palestino Edward W. Said, Orientalismo: concepciones occidentales del Oriente (Said 2002), centrada en las nociones de "discurso" y "representación", desarrolladas por Fanon y continuadas por Said, Bhabha y Spivak, principalmente. La teoría poscolonial ha generado una profunda crítica sobre cómo se ha construido el discurso occidental y las graves consecuencias económicas, políticas, sociales y culturales que ha tenido su formulación en las colonias, aspectos todos ellos recogidos en numerosos manuales al respecto (Young 2001; Loomba et al. 2005; Fernández Martínez 2006: 163-207; McLeod 2007).

Es importante, no obstante, hacer hincapié en que la alienación de los colonizados viene determinada por el propio discurso colonial europeo, que genera una separación binaria entre el colonizador y el colonizado que no sólo se impone a través de la fuerza y la coerción militar y política, sino que se apoya en construcciones culturales y simbólicas que generan nuevas categorías de oposición (Said 1996: 44; Dirks 1992). Este tipo de binarismo ha impregnado también el estudio de la presencia fenicia y griega y del colonialismo romano en el Mediterráneo, estructurado según las nociones de "civilización" (los colonizadores) y de "barbarie" (los Otros, nativos), bajo las que subyace una con- 
cepción holística de la cultura como una entidad pura e independiente del contacto con otras culturas (Friedman 1990; Barth 1992).

En el caso de la arqueología, la teoría poscolonial comenzó a tener importancia sólo en los años 90 del siglo pasado en los estudios sobre el mundo romano, preocupados por la recepción de la romanización entre los indígenas del interior europeo (Webster y Cooper 1996), desde donde se ha ido extendiendo lentamente al resto de la arqueología mediterránea (Mattingly 1996; van Dommelen 1998; Vives-Ferrándiz Sánchez 2006; Hodos 2006; Jiménez Díez 2008; Cañete Jiménez 2009; Lydon y Rizvi 2010).

Como ha sucedido con los teóricos poscoloniales, los arqueólogos se han centrado casi exclusivamente en el análisis del discurso académico en nuestra disciplina y en la representación que se ha hecho sobre los colonizadores y los colonizados en épocas pasadas (van Dommelen 2011). La inclinación de la balanza hacia lo cultural en la teoría poscolonial ha hecho, sin embargo, que se pierdan de vista las relaciones de poder que se insertaron en esos procesos, lo que les ha valido numerosas críticas (Ahmad 1994; Dirlik 1994). La falta de contextualización de los discursos poscoloniales, que no atienden a las diferencias regionales o culturales dando por hecho que en todos los lugares el proceso se desarrolló del mismo modo, ha sido otro de los grandes reproches a la teoría (Turner 1995; Huggan 1997).

No obstante, ha sido el concepto de "hibridación" definido por Bhabha el que ha levantado más voces en contra de la teoría poscolonial y de sus seguidores, que han sido definidos como una clase alta cosmopolita que poco tiene que ver con la vasta mayoría de los inmigrantes para los cuales la hibridación, la movilidad y la diferencia suponen más un desarraigo y una discriminación que una satisfacción transnacional (Parry 1987; Ahmad 1994: 207-208; Friedman 1997: 72; Hardt y Negri 2005: 175-176). La mezcla que defiende el concepto de hibridación ha sido vista, asimismo, como una invitación a la homogenización de la población que oculta la desigualdad entre los colonizadores y los colonizados y el conflicto en los contextos coloniales; entendida de esta forma, la hibridación representa más una reconciliación que una crítica al colonialismo (Steward 1999; Parry 2004: 4, 55-74; cfr. Santiago 1978; García Canclini 2003; Cañete Jiménez y Vives-Ferrándiz Sánchez 2011; Jiménez Díez 2011).

El principal problema de la teoría poscolonial es, sin embargo, su completa carencia de contacto con lo material, con lo tangible, pues trabaja con textos y discursos, no con los objetos y prácticas que rodean a las personas, que son parte inherente de su identidad y de su cotidianidad en todo tipo de situaciones, por lo que su estudio es especialmente relevante en contextos coloniales o de contacto cultural. Precisamente por este motivo, la arqueología emerge como la contribución material a dicha teoría, como el ancla de unión con la realidad cotidiana de la sociedad y de las personas inmersas en situaciones coloniales o de contacto (van Dommelen 1998: 29-35, 2006, 2011; Gosden 2001; Jiménez Díez 2011).

\section{Imaginarios coloniales y respuestas locales: el colonialismo fenicio en cuestión}

Esta importancia del estudio de la materialidad a nivel local facilita la comprensión de los procesos que tuvieron lugar entre los siglos IX y VI a.C. en el sur peninsular con la llegada de los fenicios, por un lado, y demuestra por qué la terminología utilizada para definir la presencia fenicia se corresponde más con la influencia del colonialismo europeo en nuestro modo de entender el contacto entre comunidades que con una realidad (Fig. 2). Tanto es así, que las respuestas dadas por la población del sur peninsular a la presencia oriental, al menos hasta el siglo VI a.C., no responden a la existencia de relaciones asimétricas de explotación y dominación entre colonizadores y colonizados, que es lo que define una situación colonial, según se ha planteado anteriormente, lo que nos obliga a (re) pensar las categorías de los análisis basándonos en el registro arqueológico.

Ciertamente los fenicios se establecen lejos de su lugar de origen, pero son precisamente ellos los que presentan necesidades defensivas desde el inicio, amurallando sus asentamientos, como sucede en el Castillo de Doña Blanca desde el siglo VIII a.C. (Ruiz Mata y González Rodríguez 1994: 210) o en La Fonteta a inicios del siglo VI a.C. (Gailledrat 2007: 58), no la población local, que continúa viviendo en asentamientos no fortificados hasta fines del siglo VII e inicios del siglo VI a.C. - exceptuando el poblado de Los Castillejos de Alcorrín, amurallado desde el siglo VIII a.C. e interpretado como local (Marzoli et al. 2010).

En esta misma línea cabe entender el hecho de que los asentamientos fenicios se situaran, además, en lugares costeros -Las Chorreras-, en pequeñas penínsulas - Toscanos-, o en pequeñas islas -Cerro del Villar-, guardando siempre cierta distancia con las poblaciones locales. Distancia que no se aprecia, sin embargo, en el interior de las viviendas de dichos asentamientos, donde la cerámica a mano de tradición local está presente 


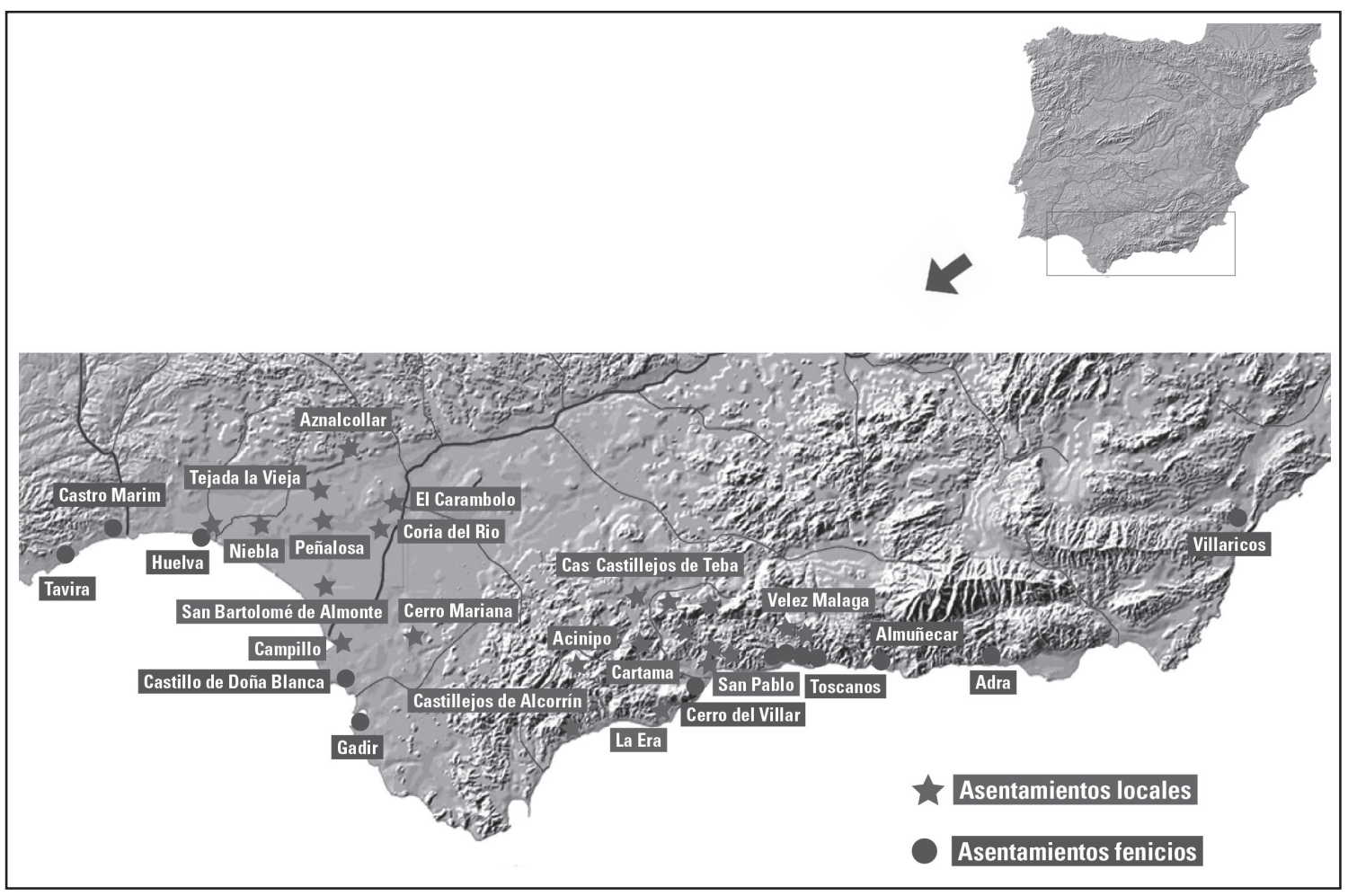

Figura 2.- Localización de los asentamientos citados en el trabajo, junto con algunos otros núcleos importantes entre los siglos IX y VII a.C. (mapa base cortesía de Jesús Arenas Esteban).

desde el inicio en Toscanos y Alarcón, en Morro de Mezquitilla, en Las Chorreras, en el Cerro del Villar y en La Rebanadilla, entre otros asentamientos fenicios, conviviendo con la cerámica fenicia en los mismos espacios (Aubet 1974, 1999; Martín Ruiz 1995; Maass-Lindemann 2002; Sánchez Sánchez-Moreno et al. 2011), lo que indica que o bien había población local viviendo en los asentamientos fenicios, o que los fenicios estaban usando esa cerámica local para determinados tipos de preparaciones y comidas, lo que en ambos casos denota un contacto con la población de los alrededores.

Por otro lado, los asentamientos fenicios del sur peninsular no se corresponden con ciudades, al menos hasta el momento y en la cronología que nos ocupa, pues en el único lugar donde se han encontrado estructuras relacionadas con el ámbito público y no con el doméstico ha sido en Toscanos, donde el "Edificio C", de gran monumentalidad y donde se halló una enorme cantidad de ánforas y de contenedores relacionados con el comercio fenicio, se ha interpretado como un almacén (Aubet 2006: 41).

Las dimensiones de los asentamientos fenicios tampoco apoyan la tesis de la existencia de verda- deras colonias fenicias, con superficies de 2 ha en el caso de Morro de Mezquitilla, de 8 ha en el Cerro del Villar y en La Fonteta, y de 10 ha en Gadir, por citar algunos ejemplos, lo que contrasta con otros asentamientos, como es el caso de Mozia, en Sicilia, que en la misma época ocupó 40 ha (Díes Cusí 2001: 87).

De forma similar, las necrópolis fenicias, aunque representen posiblemente una selección de la sociedad (plausiblemente la élite), indican que la presencia oriental hasta el siglo VII e inicios del VI a.C. fue muy reducida, ya que sólo en las necrópolis de Laurita y Puente de Noy, relacionadas con la colonia de Almuñécar, se han encontrado más de 20 tumbas (22 en concreto), en la del Cortijo de San Isidro se han hallado 12 y en el resto de cementerios el número no sobrepasa los cinco enterramientos, al contrario de las necrópolis púnicas de Jardín o Villaricos, del siglo VI al III a.C., que cuentan con más de un centenar de tumbas (Aubet 2006: 38; Martín Córdoba et al. 2006; Sánchez Sánchez-Moreno et al. 2011).

La exclusión de cultura material local en los ajuares de las necrópolis, que reflejan las costumbres de una élite exclusivamente oriental, se ha 

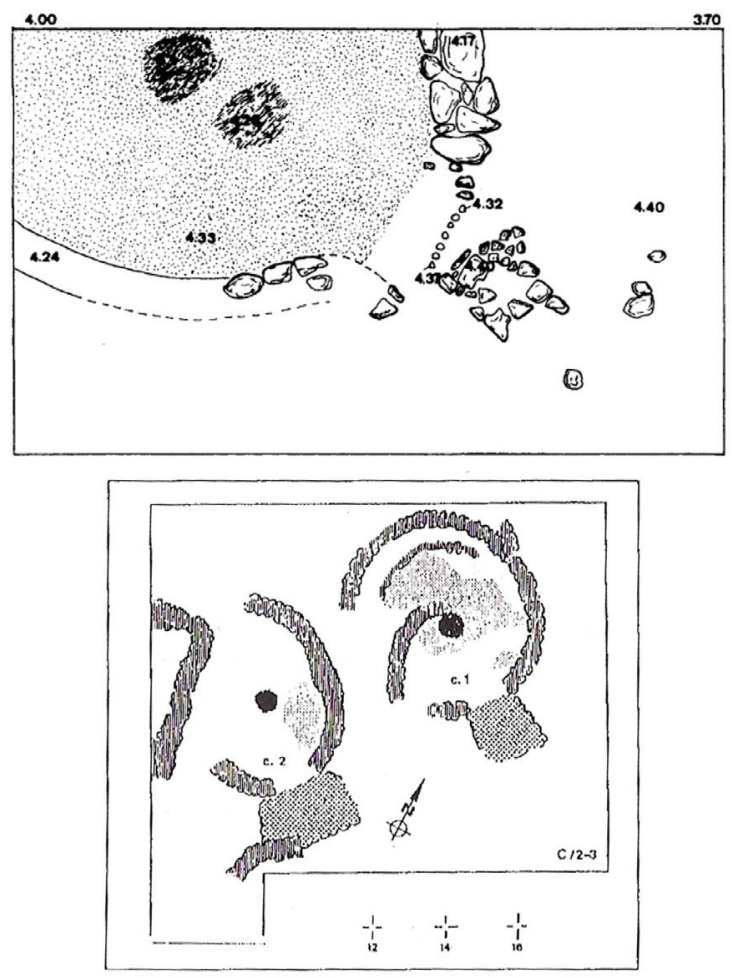

Figura 3.- Cabañas circulares del Cerro Mariana (Las Cabezas de San Juan, Sevilla) - arriba- y de Acinipo (Málaga) -abajo- (elaboración propia a partir de Izquierdo 1998; Aguayo et al. 1991).

interpretado como la legitimación del poder y la dominación colonial fenicia sobre las poblaciones locales (Delgado Hervás 2008a: 40-41). Sin embargo, el hecho de que en los rituales funerarios y en las propias necrópolis los fenicios se mantuviesen separados no significa que existiese una relación de dominación y de explotación colonial, ni siquiera lo significaría si se hubiesen mantenido completamente ajenos a la población local también en los contextos domésticos (Osborne 2008: 281). La omisión consciente de objetos y de prácticas locales podría estar más relacionada con el modo fenicio de representar el poder y, por tanto, de legitimarse frente a la comunidad que habita en los asentamientos asociados a estas necrópolis, independientemente del origen de ésta. Así, las necrópolis reflejarían unas relaciones de poder firmemente establecidas, pero no según la dualidad fenicios-indígenas, sino entre la élite (fenicia) y la población de los asentamientos de fundación oriental.

En cuanto a la población local del sur peninsular, la cultura material y las estructuras de habitación de los poblados traslucen, en términos generales, una continuidad con los modos de vida del Bronce Final, tanto en el área occidental como en la zona oriental peninsular (Fig. 3). Un ejemplo claro de esta continuidad puede apreciarse en la aparición y uso de las cerámicas grises "orientalizantes" o monocromas en el sur peninsular. La producción de este tipo de cerámica acontece entre los siglos VIII y VI a.C. y se detecta en las zonas levantina, occidental y meridional de la Península Ibérica, asociada al contacto fenicio (Vallejo Sánchez 2005 con bibliografía anterior). Independientemente del debate en torno al origen de la producción de esta cerámica, fenicia o local, las cerámicas grises reproducen formas locales, principalmente abiertas, como las del Bronce Final; y denotan una estética local, como demuestran las superficies bruñidas y las decoraciones, cuando las hay (Vallejo Sánchez 2005: 1161-1163), lo que indica que estas cerámicas responden a un modo de servir y de consumir los alimentos fundamentalmente local (Fig. 4). En relación a los contextos domésticos, tanto en los asentamientos formados por cabañas, como en aquellos donde se introducen cambios como las estructuras rectangulares, es la cerámica gris la más demandada generalmente y no la de engobe rojo (Ruiz Mata y González Rodríguez 1994: 221; Sanna 2009). En este sentido, cobra importancia el hecho de que sea la cerámica gris la que sustituya a la cerámica a mano a partir del siglo VII a.C. (exceptuando las ollas, que son sustituidas sólo a partir del siglo VI a.C.) (Vallejo Sánchez 2005: 1167), y no la cerámica fenicia.

En los contextos rituales, como los de la necrópolis de La Joya (Huelva) o los de Los Alcores (Sevilla), donde el estudio de los materiales ha potenciado las asunciones sobre el "orientalizante" peninsular y la existencia de unas élites profundamente "orientalizadas" (Torres Ortiz 2002, 2005 con bibliografía anterior), se puede detectar ya desde el siglo VIII a.C. que, aunque se adoptan los jarros metálicos y de alabastro fenicios, para el consumo ritual se utilizan los cuencos bruñidos, grises o de tipo Medellín de tradición local (Jiménez Flores et al. 2005: 689). Así pues, dentro de un ritual en el que aparecen las ánforas y los objetos metálicos fenicios, la élite local supuestamente "orientalizada", lejos de asumir los rituales fenicios (tampoco hay indicios del banquete funerario oriental en el exterior de las tumbas), continúa consumiendo las ofrendas en la vajilla de tradición local.

Interesante es también el hecho de que en las viviendas de los asentamientos fenicios se hayan encontrado los vasos de cerámica gris, junto con cerámicas a mano, como en Toscanos y Alarcón, en Morro de Mezquitilla, en Las Chorreras, en el 


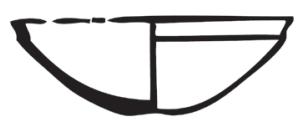

1

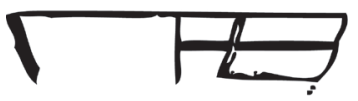

2

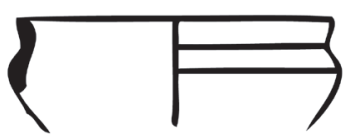

3

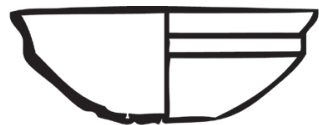

4
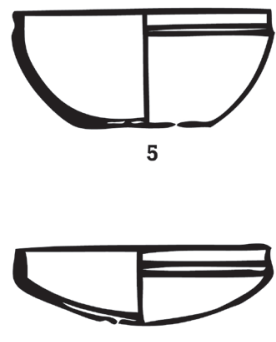

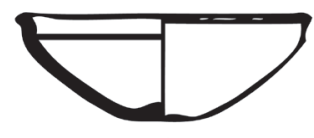

7

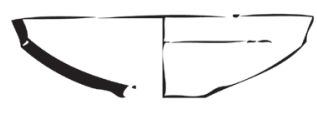

8

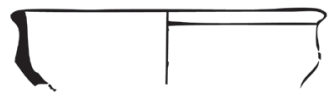

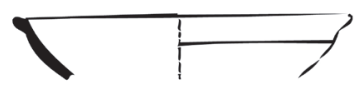

10
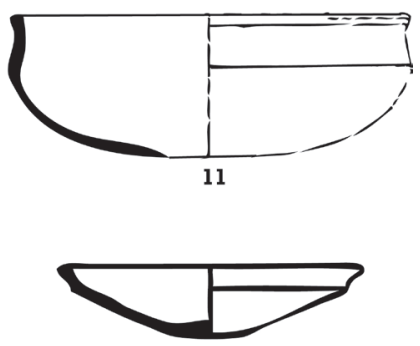

12

Figura 4.- Cerámicas a mano bruñidas (1-6) y cerámicas grises monocromas (7-12) (elaboración propia a partir de Torres Ortiz 2002: 127, 139; Vallejo Sánchez 2005: 1157).

Cerro del Villar y en La Rebanadilla (Aubet 1974, 1999; Martín Ruiz 1995; Maass-Lindemann 2002; Sánchez Sánchez-Moreno et al. 2011). Este tipo de cerámica, curiosamente, llegó a sustituir a los platos y cuencos de engobe rojo en algunos asentamientos como el Cerro del Alarcón (Maass-Lindemann 2002: 199), lo que indica la persistencia de las tradiciones culinarias locales, aunque en convivencia con las foráneas, incluso en contextos orientales.

De forma similar, y en términos generales, la población local continuó viviendo en los mismos asentamientos de cabañas anteriores a la llegada fenicia, al menos hasta el siglo VI a.C. (Ruiz Mata y González Rodríguez 1994; Izquierdo de Montes 1998; Fernández Jurado 2003: 37-41; López Pardo y Suárez Padilla 2003), si bien es cierto que muchos podrían pasar a vivir en los propios establecimientos fenicios, como podría demostrar la existencia de la cultura material local antes comentada. La adopción, aunque muy limitada, como se deduce del registro arqueológico, de nuevas técnicas constructivas que hacen referencia a modelos fenicios, coincide en la mayoría de los casos con los grandes núcleos de población que ya se definían como centros de poder desde el Bronce Final (Ruiz Mata y González Rodríguez 1994; Ruiz Mata et al. 1998). En muchos de estos poblados, además, la introducción de estructuras rectangulares convive con las cabañas circulares (Ruiz Mata y González Rodríguez 1994; López Pardo y Suárez Padilla 2003; García Alfonso 2007), lo que indica que sólo una parte de la población, presumiblemente la élite, está adoptando las técnicas fenicias. Cabe añadir, en este sentido, que sólo en el caso de La Era se ha documentado un edificio rectangular en torno a un patio central, exceptuando las estructuras interpretadas como templos en Montemolín, en el siglo VIII a.C. y en la Algaida y Cancho Roano, en el siglo VI a.C. (Torres Ortiz 2002: 301-303; López Pardo y Suárez Padilla 2003: 81). En los demás asentamientos de estas características, las estructuras rectangulares no responden a patrones de uso, ni de concepción y distribución del espacio, ni de movilidad fenicios, sino locales, sin prácticamente compartimentación interna, como sucede en las cabañas contemporáneas (Izquierdo de Montes 1998; Díes Cusí 2001: 97-98).

Dicho lo cual, cabe incidir en que la violencia y las relaciones de poder siempre existieron. En los poblados de la Andalucía mediterránea, como sucedía en el caso de los fenicios, las relaciones de dominación y explotación y la división social se estarían estableciendo entre el linaje o la élite que ostentaba el poder y el resto de la población, fuese ésta de origen fenicio o de origen local. Sin embargo, no se constituirían sobre una base binaria colonial que, por otro lado y después de dos siglos, sería difícil de distinguir (¿quién es "fenicio" y quién "local"?). Durante todo el contacto, además, tanto fenicios como locales se caracterizarían por identidades mucho más complejas, imbricadas en cuestiones étnicas, sociales, de género o de edad, que serían la base para establecer quién pertenecía a los grupos de poder y quién no. La población subalterna, tanto de los asentamientos en origen fenicios como de los poblados en ori- 
gen locales, estuvo desde el principio sometida al poder de la élite que gobernaba, estuviese esta formada por la clase alta fenicia, por la local o por la mezcla de ambas. De este modo, la agencia subalterna y la creatividad de la población dominada es muy limitada. Tanto es así, que sólo en las tumbas "principescas" se encuentran los objetos suntuosos fenicios (o en mayor número), mientras que el resto de la población o no se enterraba o se enterraba en las denominadas "necrópolis planas" y "necrópolis rurales" (Torres 2005). Del mismo modo, la convivencia de la cerámica de servicio de mesa fenicia y local en los asentamientos de origen fenicio, podría estar relacionada con las diferencias sociales entre la élite fenicia y el resto de la población, fenicia y local, que compartían clase social y, por tanto, vajilla y comida (Delgado Hervás, 2008b: 169).

Sólo a partir del siglo VII y, sobre todo, a inicios del siglo VI a.C. (doscientos años después del inicio del contacto), se observa una reorganización de la geografía poblacional en el sur peninsular, donde tanto los poblados locales como las colonias fenicias se amurallan, caso de Cerro del Cabrero o de Castillejos de Teba, ambos en Málaga (López Pardo y Suárez Padilla 2003: 81); o de Toscanos y Alarcón (Schubart 2000: 264; Schubart 2002: 134), y de Malaka, en la misma provincia (Suárez Padilla et al. 2001: 118-119), o de la Fonteta en Alicante (Gailledrat 2007: 58). A ello se añade la fundación de nuevos enclaves en zonas de gran interés agrícola, tanto por parte de las poblaciones locales como por parte de la población de los asentamientos de origen fenicio; los primeros indicios de conflictos bélicos, como parece indicar el caso de Castellón de Gobantes (Málaga) y la emergencia de élites guerreras (García Alfonso 2002: 141); y la aparición de nuevos asentamientos, como Villaricos en Almería (López Castro 2007). La búsqueda de nuevas tierras de cultivo genera una rivalidad por el control de los recursos entre las poblaciones del sur peninsular, lo que nos habla de un nuevo tipo de relaciones de poder que se están estableciendo y que se desarrollarán en los siglos posteriores, ésta vez sí de corte más violento y competitivo y quizá sí, entonces, colonial.

\section{Consideraciones finales}

El colonialismo europeo y la construcción de la identidad europea han influenciado enormemente la comprensión de los procesos de contacto cultural y colonial en el Mediterráneo antiguo. Como consecuencia, la cultura griega y la romana han sido potenciadas en detrimento de la cultura orien- tal, que ha quedado en un plano muy secundario hasta que se ha insertado en los parámetros de la "colonización" griega y de la conquista romana. Tanto es así que la presencia fenicia en el Mediterráneo y, sobre todo, en el sur peninsular, ha sido definida con los mismos términos que la griega y la romana, ignorando las diferencias de cada proceso.

Asimismo, imbuidos en el discurso europeo de "civilización" frente a la "barbarie" de la población colonizada, los estudios sobre el colonialismo antiguo han reproducido fielmente la imagen de la "misión civilizadora", donde los fenicios, los griegos, los cartagineses (éstos en menor medida) y los romanos llevaban la cultura a las comunidades bárbaras y retrasadas, introduciéndose nociones de factura imperial como "orientalizante", "helenización" o "romanización" en los análisis arqueológicos e históricos, que han sido escasamente cuestionados en el caso oriental. En este sentido, el término "orientalizante" debería ser excluido de los análisis arqueológicos por haber magnificado el papel de los fenicios, cuando la mayoría de los asentamientos y de la cultura material local mantienen una continuidad, y por haber silenciado las respuestas de la población local, lo que demuestra el enorme impacto que sigue teniendo el colonialismo europeo en el estudio de los fenómenos coloniales y de contacto en el Mediterráneo.

La crítica de estos discursos y de las desastrosas consecuencias sociales, culturales, económicas y políticas que han tenido en las colonias, se ha reflejado en numerosas iniciativas políticas desde los años 20 del siglo pasado y en las denuncias de un nada desdeñable número de activistas de las antiguas ex-colonias. La teoría poscolonial sigue la estela de estas críticas al discurso colonial europeo, aunque con un enfoque que excluye de sus análisis la materialidad, de forma que la arqueología se presenta como la contribución clave para el estudio de la realidad en contextos coloniales o de contacto entre diferentes culturas. A este respecto, el análisis conjunto de los asentamientos fenicios y de sus necrópolis, y de los asentamientos locales y de las necrópolis conocidas, demuestra que el período comprendido entre los siglos IX y VII/VI a.C. no respondió a un colonialismo fenicio, sino más bien a un contacto cultural entre diferentes poblaciones que se desarrolló de modos diferentes dependiendo del contexto local en el que se insertó, pero que en ningún caso se correspondió con una dominación de la población por parte de los fenicios, lo que fundamenta aún más la crítica contra el uso del término orientalizante y el de colonia para designar los asentamientos de fundación oriental en el sur peninsular. 


\section{Agradecimientos}

Estoy en deuda con Peter van Dommelen, Alicia Jiménez y Jaime Vives-Ferrándiz por sus comentarios al borrador de este trabajo y por los largos debates mantenidos en torno a la teoría poscolonial y al contexto fenicio del sur peninsular. Agradezco, asimismo, los comentarios y sugerencias de los/as evaluadores/as de este trabajo, que me han ayudado a mejorar la presente versión de este texto. Cualquier error existente, sin embargo, es sólo responsabilidad mía. Este artículo es parte de la investigación llevada a cabo en mi tesis doctoral, financiada por el Ministerio de Educación dentro del programa de Formación del Profesorado Universitario (FPU).

\section{REFERENCIAS BIBLIOGRÁFICAS}

Abdel-MaleK, A. (1963): Orientalism in crisis. Diogenes, 44: 105-123.

Aguayo, P.; Carrilero, M.; Martínez, G. (1991): La presencia fenicia y el proceso de aculturación de las comunidades del Bronce Final de la depresión de Ronda (Málaga). Atti del II Congresso Internazionale di Studi Fenici e Punici (Roma, 1987). Consiglio Nazionale della Ricerca, Roma: 559-571.

Ahmad, A. (1994): In theory: classes, nations, literatures. Verso, Londres.

Almagro Gorbea, M. (1991): El mundo orientalizante en la Península Ibérica. Atti del II Congresso Internazionale di Studi Fenici e Punici (Roma, 9-14 novembre 1987). Consiglio Nazionale della Ricerca, Roma: 573-599.

Alvar Ezquerra, M.; Nieto Jiménez, L. (2007): Nuevo tesoro lexicográfico del español (s. XIV - 1726). Arco Libros, Madrid.

De Angelis, F. (2009): Colonies and colonization. The Oxford Handbook of Hellenic Studies (G. Boys-Stones, B. Graziosi, y P. Vasunia, eds.), Oxford University Press, Oxford: 48-64.

Arteaga Matute, O. (1995): Paradigmas historicistas de la civilización occidental. Los fenicios en las costas mediterráneas. Spal, 4: 131-171.

Aubet, M.E. (1974): Excavaciones en Las Chorreras, Mezquitilla (Málaga). Pyrenae: revista de prehistòria $i$ antiguitat de la Mediterrània Occidental, 10: 79-108.

Aubet, M.E. (1997): Tiro y las colonias fenicias de Occidente. Crítica, Barcelona.

Aubet, M.E. (1999): Los materiales. Cerro del Villar - I. El asentamiento en la desembocadura del río Guadalhorce y su interacción con el hinterland (Aubet, M.E.; Carmona, P.; Curià, E.; Fernández Cantos, A.; Párraga, M., eds.), Junta de Andalucía. Consejería de Cultura, Sevilla: 86-127.

Aubet, M.E. (2005): El 'Orientalizante': un fenómeno de contacto entre sociedades desiguales. En Celestino Pérez y Jiménez Ávila 2005: 117-128.

Aubet, M.E. (2006): El sistema colonial fenicio y sus pautas de organización. Mainake, XXVIII: 35-47.

Aubet, M.E.; Delgado Hervás, A.M. (2003): La colonia fenicia del Cerro del Villar y su territorio. Ecohistoria del paisaje agrario: la agricultura fenicio-púnica en el Mediterráneo (C. Gómez Bellard, ed.), Universitat de València, València: 57-74.

BARTH, F. (1992): Towards greater naturalism in conceptualizing societies. Conceptualizing society (A. Kuper, ed.), Routledge, Londres: 17-33.

Bispham, E. (2006): Coloniam deducere: How Roman was Roman colonization during the Middle Republic? Greek and Roman Colonisation: Origins, Ideologies and Interactions (G. Bradley; J. P. Wilson, eds.), Classical Press Wales, Cardiff: 73-160.

BlÁzQuez, J.M. (1992): Fenicios, griegos y cartagineses en Occidente. Cátedra, Madrid.

BoArdman, J. (1980): The Greeks overseas. Thames and Hudson, Londres.

CAÑETE JimÉnEz, C. (2009): El origen africano de los Íberos: una perspectiva historiográfica. Universidad de Málaga, Málaga.

Cañete Jiménez, C.; Vives-Ferrándiz SÁnchez, J. (2011): “Almost the same”: dynamic domination and hybrid contexts in Iron Age Lixus, Larache, Morocco. World Archaeology, 43(1): 124-143.

Celestino Pérez, S. (2008): Tartessos. De Iberia a Hispania (F. Gracia Alonso, ed.), Ariel, Barcelona: 93-345.

Celestino Pérez, S.; Jiménez Ávila, J. (eds.) (2005): El Período Orientalizante: Actas del III Simposio Internacional de Arqueología de Mérida: Protohistoria del Mediterráneo Occidental. Consejo Superior de Investigaciones Científicas, Madrid. 
Delgado Hervás, A.M. (2008a): 'Colonialismos' fenicios en el sur de Iberia: historias precedentes y modelos de contacto. De Tartessos a Manila: siete estudios coloniales y poscoloniales (G. Cano; A. M. Delgado Hervás, eds.), Universitat de València, València: 19-49.

Delgado Hervás, A. M. (2008b): Alimentos, Poder e Identidad En Las Comunidades Fenicias Occidentales. Cuadernos de Prehistoria y de Arqueología de la Universidad de Granada, 18: 163-88.

Díes Cusí, E. (2001): La influencia de la arquitectura fenicia en las arquitecturas indígenas de la Península Ibérica (ss. VIII-VII). En Ruiz Mata y Celestino Pérez 2001: 69-122.

Dirks, N.B. (1992): Introduction: colonialism and culture. Colonialism and culture (N.B. Dirks, ed.), University of Michigan Press, Michigan: 1-25.

Dirlik, A. (1994): The postcolonial aura: Third World criticism in the age of global capitalism. Critical Inquiry, 20(2): 328-356.

Evans, J.; Boswell, D. (eds.) (1999): Representing the nation. A reader: histories, heritage and museums. Routledge, Londres.

FernÁNdez Jurado, J. (2003): Indígenas y fenicios en Huelva. Huelva Arqueológica, 18: 33-54.

Fernández Martínez, V.M. (2006): Una Arqueología Crítica: Ciencia, Ética y Política en la Construcción del Pasado. Crítica, Barcelona.

Friedman, J. (1990): Notes on culture and identity in imperial worlds. Religion and religious practice in the Seleucid Kingdom (P. Bilde, T. Engberg-Pedersen, L. Hannestad, J. Zahle, eds.), Aarhus University Press, Aarhus: 14-39.

Friedman, J. (1992): The past in the future: history and the politics of identity. American Anthropologist, 94(4): $837-859$.

FRIEDMAN, J. (1997): Global crisis, the struggle for cultural identity and intellectual porkbarrelling: cosmopolitans versus locals, ethnics, and nationals in an era of de-hegemonization. Debating Cultural Hybridity: Multi-Cultural Identities and the Politics of Anti-Racism (P. Werbner y T. Modood, eds.), Zed Books, Londres: 70-89.

GaIlledrat, E. (2007): La stratigraphie. L'établissement protohistorique de La Fonteta (fin VIIIe siècle av. J.-C.) (P. Rouillard, E. Gailledrat y F. Sala Sellés, eds.), Casa de Velázquez, Madrid: 23-97.

García Alfonso, E., (2002): La primera historia de Málaga: la colonización fenicia arcaica. Servicio de Publicaciones de la Universidad de Málaga, Málaga.

García Alfonso, E. (2007): En la orilla de Tartessos: fenicios e indígenas en tierras malagueñas (siglos VIII-VI a.C.). Fundación Málaga, Málaga.

García Canclini, N. (2003): Noticias recientes sobre la hibridación. Trans: Revista Transcultural de Música, 7. [URL: http://www.sibetrans.com/trans/a209/noticias-recientes-sobre-la-hibridacion]. Acceso el 07/06/2012.

Gillis, J.R. (ed.) (1994): Commemorations: the politics of national identity. Princeton University Press, Princeton.

González de Canales, F.; Serrano Pichardo, L.; Llampart Gómez, J. (2004): El emporio fenicio precolonial de Huelva (ca. 904-770 a.C). Biblioteca Nueva, Madrid.

González Prats, A. (1999): La Fonteta, 1996-1998. El emporio fenicio de la desembocadura del río Segura. Guía de la Exposición Monográfica con motivo del II Seminario Internacional sobre Temas Fenicios. Alicante.

González Ruibal, A. (2008): Postpolitical colonialism. Journal of Mediterranean Archaeology, 21(2): $285-288$.

GonzÁlez Ruibal, A. (2010): Colonialism and European archaeology. Handbook of Postcolonial Archaeology (J. Lydon y U. Rizvi, eds.). Left Coast Press, Walnut Creek: 37-47.

GonzÁlez Wagner, C. (1993): Aspectos socioeconómicos de la expansión fenicia en Occidente: el intercambio desigual y la colonización agrícola. Estudis d'Història econòmica, I: 13-37.

GonzÁlez WaGner, C. (2007): El barco negro en la costa. Reflexiones sobre el miedo y la colonización fenicia en la tierra de Tarsis. Gerión, Extra: 121-131.

Gosden, C. (2001): Postcolonial archaeology. Issues of Culture, Identity, and Knowledge. Archaeological Theory Today (I. Hodder, ed.), Polity Press and Blackwell, Cambridge: 241-261.

Gosden, C. (2004): Archaeology and colonialism: culture contact from 5000 BC to the present. Cambridge University Press, Cambridge.

Grosfoguel, R. (2007): The epistemic decolonial turn. Cultural Studies, 21(2/3): 211-223.

HALl, E. (1989): Inventing the barbarian: Greek self-definition through tragedy. Oxford University Press, Oxford. Hardt, M.; Negri, A. (2005): Imperio. Paidós Ibérica, Barcelona. 
Hingley, R. (2000): Roman officers and English Gentlemen: the Imperial Origins of Roman Archaeology. Routledge, Londres.

Hingley, R. (ed.) (2001): Images of Rome: Perceptions of Ancient Rome in Europe and the U.S. in the Modern Age. Journal of Roman Archaeology, 44.

Hodos, T. (2006): Local Responses to Colonization in the Iron Age Mediterranean. Routledge, Londres.

Horvath, R.J. (1972): A definition of colonialism. Current Anthropology, 13(1): 45-57.

Huggan, G. (1997): The Neocolonialism of Postcolonialism: a Cautionary Note. Links \& Letters, 4: 19-24.

IzQuierdo DE Montes, R. (1998): La cabaña circular en el mundo tartésico. Consideraciones sobre su uso como indicador étnico. Zephyrus, 51: 277-288.

Jiménez Ávila, J. (2002): La toreútica orientalizante en la Península Ibérica. Real Academia de la Historia, Madrid.

JimÉNEZ DíEz, A. (2008): Imagines hibridae: una aproximación postcolonialista al estudio de las necrópolis de la Bética. Consejo Superior de Investigaciones Científicas, Madrid.

Jiménez DíEz, A. (2011): Pure hybridism: Late iron Age sculpture in southern Iberia. World Archaeology, 43(1): $102-123$.

Jiménez Flores, A.M.; García Fernández, F.J.; Camacho Moreno, M. (2005): In vino humanitas: el vino y su función socio-ideológica en el mundo orientalizante. En Celestino Pérez y Jiménez Ávila 2005: 683-691.

Johnson, R.R. (1988): Hellas in Hesperia. Paths from ancient Greece (C. G. Thomas, ed.), E.J. Brill, Leiden: $140-167$.

Leoussi, A.S. (1998): Nationalism and Classicism. Macmillan, Londres.

Loomba, A.; Kaul, S.; Bunzl, M.; Burton, A.; Esty, J. (eds.) (2005): Postcolonial Studies and Beyond. Duke University Press, Durham, NC.

López Castro, J.L. (2007): La ciudad fenicia de Baria. Investigaciones 1987-2003. Actas de las Jornadas sobre la Zona Arqueológica de Villaricos, 26, 27 y 28 de enero de 2005, Almería. Consejería de Cultura. Dirección General de Bienes Culturales, Sevilla: 19-39.

López Pardo, F.; SuÁrez Padilla, J. (2003): Aproximación al conocimiento del paleoambiete, poblamiento y aprovechamiento de los recursos durante el primer milenio a.C. en el litoral occidental de Málaga. Ecohistoria del paisaje agrario: la agricultura fenicio-púnica en el Mediterráneo (C. Gómez Bellard, ed.), Universitat de València, València: 75-92.

Lydon, J.; Rizvi, U. (eds.) (2010): Handbook of Postcolonial Archaeology. Left Coast Press, Walnut Creek, CA.

MaAss-Lindemann, G. (2002): Los hallazgos fenicios del Cerro del Alarcón. Cuadernos de Arqueología Mediterránea, 8: 189-243.

Malkin, I. (2003): Networks and the emergence of Greek identity. Mediterranean Historical Review, 18 : 56-74.

Malkin, I. (2004): Postcolonial Concepts and Ancient Greek Colonization. MLQ: Modern Language Quarterly, 65(3): 341-364.

Martín Córdoba, E.; Ramírez Sánchez, J.; Ruescas Pareja, V.; Recio Ruiz, A. (2006): Necrópolis fenicias de los siglos VIII-VII a.C. en la desembocadura del río Vélez (Vélez-Málaga, Málaga). Mainake, XXVIII: $303-331$.

Martín Ruiz, J.A. (1995): Indicadores arqueológicos de la presencia indígena en las comunidades fenicias de Andalucía. Mainake, XVII-XVIII: 73-90.

Marzoli, D.; López Pardo, F.; Suárez Padilla, J.; González Wagner, C.; Mielke, D.P.; León Martín, C.; Ruiz Cabrero, L.; Thiemeyer, H.; Torres Ortiz, M. (2010): Los inicios del urbanismo en las sociedades autóctonas localizadas en el entorno del Estrecho de Gibraltar: investigaciones en Los Castillejos de Alcorrín y su territorio (Manilva, Málaga). Menga: Revista de Prehistoria de Andalucía, 1: 152-182.

Mattingly, D.J. (1996): From one imperialism to another. Imperialism in the Maghreb. Roman imperialism: postcolonial perspectives (J. Webster y N. Cooper, eds.), Leicester Archaeology monographs, 3: 49-70.

Mattingly, D.J. (2011): Imperialism, Power, and Identity: Experiencing the Roman Empire. Princeton University Press, Princeton.

McLeod, J. (ed.) (2007): The Routledge Companion to Postcolonial Studies. Routledge, Londres.

Mignolo, W. (2008): La opción descolonial. Letral, 1: 4-22.

Moreno Arrastio, F.J. (1999): Conflictos y perspectivas en el período precolonial tartésico. Gerión, 17: 149-177.

Morris, I. (1994): Archaeologies of Greece. Classical Greece: ancient histories and modern archaeologies (I. Morris, ed.), Cambridge University Press, Cambridge: 9-47. 
Niemeyer, H.-G. (1990): The Phoenicians in the Mediterranean: a non-Greek model for expansion and settlement in Antiquity. Greek Colonists and Native Populations (Proceedings of the first Australian congress of Classical Archaeology held in honour of Emeritus Professor A.D. Trendall, Sydney 9-14 July 1985) (J.P. Descoeudres, ed.), Clarendon Press, Oxford: 469-489.

Osborne, R. (1998): Early Greek colonisation? The nature of Greek settlement in the West. Archaic Greece: New Approaches and New Evidence (N. Fisher; H. van Wees, eds.), Duckworth, Londres: 251-270.

Osborne, R. (2008): Colonial cancer. Journal of Mediterranean Archaeology, 21(2): 281-284.

Owen, S. (2005): Analogy, archaeology and archaic colonization. Ancient Colonisations: Analogy, Similarity and Difference (H. R. Hurst; S. Owen, eds.), Duckworth, Londres: 115-139.

PARry, B. (1987): Problems in current discourse theory. Oxford Literary Review, 9: 27-58.

PArry, B. (2004): Postcolonial Studies: a Materialist Critique. Routledge, Londres.

Purcell, N. (2006): Orientalizing: five historical questions. Debating Orientalization: multidisciplinary approaches to change in the ancient Mediterranean (C. Riva; N. Vella, eds.), Equinox Monographs in Mediterranean Archaeology, Londres: 21-30.

Richard, C.J. (1995): The founders and the Classics: Greece, Rome and the American Enlightenment. Harvard University Press, Harvard.

Ridgway, D. (1992): The first Western Greeks. Cambridge University Press, Cambridge.

Rowlands, M. (1998): The Archaeology of Colonialism. Social Transformations in Archaeology. Global and Local Perspectives (K. Kristiansen; M. Rowlands, eds.), Routledge, Londres.

Ruiz Mata, D.; Celestino Pérez, S. (eds.) (2001): Arquitectura oriental y orientalizante en la Península Ibérica. Consejo Superior de Investigaciones Científicas, Madrid.

Ruiz Mata, D.; GonzÁlez Rodríguez, R. (1994): Consideraciones sobre asentamientos rurales y cerámicas orientalizantes en la campiña gaditana. Spal, 3: 209-256.

Ruiz Mata, D.; Niveau de Villedary, A.; Vallejo Sánchez, J.I. (1998): La ciudad tartésica-turdetana. Actas del Congreso Internacional Los Íberos. Príncipes de Occidente. Estructuras de poder en la sociedad ibérica. Fundación La Caixa, Barcelona: 65-82.

SAID, E.W. (1996): Cultura e imperialismo. Anagrama, Barcelona.

SAID, E.W. (2002 [1978]): Orientalismo. Debate, Madrid.

Sánchez SÁnchez-Moreno, V.M.; Galindo San José, L.; Juzgado Navarro, M.; Dumas Peñuelas, M.; DomínGUEz PÉREZ, J.C. (2011): La desembocadura del Guadalhorce en los siglos IX y VIII a.C. y su relación con el Mediterráneo. Gadir y el Círculo del Estrecho revisados: propuestas de la arqueología desde un enfoque social (J. C. Domínguez Pérez, ed.), Servicio de Publicaciones de la Universidad de Cádiz, Cádiz: 187-197.

SANNA, C. (2009): La cerámica gris orientalizante entre tradición e innovación: el caso de Ronda La Vieja (Acinipo) (Ronda, Málaga). Arqueología y Territorio, 6: 151-164.

Santiago, S. (1978): O entre-lugar do discurso latino-americano. Uma literatura nos tropicos: Ensaios sobre dependencia cultural. Editora Perspectiva, São Paulo: 11-28.

Schubart, H. (2000): Alarcón. El yacimiento fenicio y las fortificaciones en la cima de Toscanos. Fenicios y Territorio: Actas del II Seminario Internacional sobre temas fenicios (A. González Prats, ed.), Instituto Alicantino de Cultura Juan Gil-Albert/Direcció General d'Ensenyaments Universitaris i Investigació, Alicante: 263-294.

Schubart, H. (2002): Toscanos y Alarcón: el asentamiento fenicio en la desembocadura del río Vélez. Cuadernos de Arqueología Mediterránea, 8: 1-251.

Shanks, M. (1996): Classical archeology of Greece. Routledge, Londres.

SpIVAK, G.C. (1990): The post-colonial critic. Interviews, strategies, dialogues. Routledge, Nueva York.

Stein, G.J. (2002): Colonies without Colonialism: A Trade Diaspora Model of Fourth Millennium B.C. Mesopotamian Enclaves in Anatolia. The archaeology of colonialism (C. L. Lyons; J. K. Papadopoulos, eds.), Getty Research Institute, Los Angeles, CA: 27-64.

SteWARD, C. (1999): Syncretism and its synonyms: reflections on cultural mixture. Diacritics, 29(3): 40-62.

Suárez Padilla, J.; Navarro, I.; Fernández Rodríguez, L.E.; Mayorga, J.; Cisneros, M.I.; Wulff Alonso, F.; Cruz Andreotti, G.; Martínez Maza, C. (2001): Consideraciones acerca de los procesos de interacción entre indígenas, fenicios y griegos en Málaga. Aportaciones de la Arqueología de Urgencia. Comercio y comerciantes en la Historia Antigua de Málaga (siglos VIII a.C.-711 d.C.) (F. Wulff Alonso; G. Cruz Andreotti; C. Martínez Maza eds.), Diputación de Málaga, Málaga: 99-142. 
Torres Ortiz, M. (2002): Tartessos. Real Academia de la Historia, Madrid.

Torres Ortiz, M. (2005): Las necrópolis orientalizantes del Sudoeste de la Península Ibérica. En Celestino Pérez y Jiménez Ávila 2005: 423-440.

Turner, L. (1995): Consuming colonialism. Critique of Anthropology, 15(2): 203-212.

VAllejo SÁnchez, J.I. (2005): Las cerámicas grises orientalizantes de la Península Ibérica: una nueva lectura de la tradición alfarera indígena. En Celestino Pérez y Jiménez Ávila 2005: 1149-1172.

van Dommelen, P. (1997a): Colonial constructs: colonialism and archaeology in the Mediterranean. World Archaeology, 28(3): 305-323.

van Dommelen, P. (1997b): Some reflections on urbanization in a colonial context. West central Sardinia in the 7th to 5th century BC. Urbanization in the Mediterranean in the 9th to the 6th centuries BC. Proceedings of the International Symposium in Copenhagen (May 1994) (= Acta Hyperborea 7, 243-278) (H. Damgaard Andersen; H. Horsnaes; S. Houby-Nielsen, eds.). Museum Tusculanum Press, Copenhagen: 243-278.

VAN DOMMELEN, P. (1998): On colonial grounds: a comparative study of colonialism and rural settlement in first millennium BC west central Sardinia. Faculty of Archaeology, Leiden University, Leiden.

van Dommelen, P. (2005): Colonial interactions and hybrid practices: Phoenician and Carthaginian settlement in the ancient Mediterranean. The archaeology of colonial encounters: comparative perspectives (G. J. Stein, ed.), School of American Research Advanced Seminar Series, Santa Fe: 109-141.

van Dommelen, P. (2006): Colonial matters. Material culture and postcolonial theory in colonial situations. Handbook of material culture (C. Tilley, W. Keane, S. Kuechler, M. Rowlands, P. Spyer, eds.), Sage, Londres: 104-124.

VAN Dommelen, P. (2011): Postcolonial archaeologies between discourse and practice. World Archaeology, 43(1): $1-6$.

van Dommelen, P. (2012): Colonialism and Migration in the Ancient Mediterranean. Annual Review of Anthropo$\log y, 41: 393-409$.

Vella, N. (1996): Elusive Phoenicians. Antiquity, 70(268): 245-250.

Vives-Ferrándiz SÁnchez, J. (2006): Negociando encuentros: situaciones coloniales e intercambios en la costa oriental de la Península Ibérica (ss. VIII-VI a.C.). Bellaterra, Barcelona.

Webster, J.; CoOPer, N. (1996): Roman imperialism: post-colonial perspectives. Leicester Archaeology monographs, 3 .

Woolf, G. (1997): Beyond Romans and natives. World Archaeology, 28(3): 337-350.

Young, R.J.C. (2001): Postcolonialism: an historical introduction. Blackwell, Oxford. 